\title{
Two Time-Scale Content Caching and User Association in 5G Heterogeneous Networks
}

\author{
Jeongho Kwak $\dagger$, Long Bao Le $\ddagger$ and Xianbin Wang $₫$ \\ $\dagger$ School of Computer Science \& Statistics, Trinity College Dublin, Ireland \\ $\ddagger$ INRS-EMT, University of Quebec, Canada \\ \Electrical and Computer Engineering, Western University, Canada \\ E-mail: jeongho.kwak@tcd.ie, long.le@emt.inrs.ca, xianbin.wang@uwo.ca
}

\begin{abstract}
In this paper, we develop a content caching and flowlevel BS-user association framework in a network environment with the spatial variation of content popularity. Because the studied content caching and BS-user association functions are tightly intertwined with each other, and their decision time scales can be very different in practice, our design considers the time-scale separation of these network functions to tackle and develop the BS-user association and content caching policies. Specifically, we propose an optimal BS-user association algorithm, namely $O p t U A$, operating in the short time scale for a given content caching solution, and a greedy content caching algorithm, namely $G C C$, operating in the long time scale. The GCC algorithm exploits the submodularity characteristics of the objective function which ensures that the GCC algorithm achieves a constant fraction of the optimal performance for most feasible caching sets. Via extensive numerical studies in heterogeneous cellular networks, we demonstrate that proposed OptUA and GCC algorithms outperform other algorithms which do not consider spatial variations of content popularity in terms of average end-to-end delay per content request and average system load per content at each BS.
\end{abstract}

\section{INTRODUCTION}

As the demand for mobile video data increases exponentially in recent years ${ }^{1}$, efficient content caching plays a vital role in future $5 \mathrm{G}$ wireless networks. Many content caching algorithms [2]-[5], which cache popular contents at network edges closer to end users, have been proposed to significantly alleviate the traffic load in the core network and improve endto-end service latency.

However, most previous works have not captured the spatiotemporal dynamics of content requests. In fact, content popularity patterns can vary over the time and space in practice. For example, the Yankees' baseball game is likely to be more popular in the Manhattan area at specific time zone than in other areas and/or different time zones. These spatio-temporal dynamics of content popularity patterns are very similar to the variations of spatial traffic patterns over time [6] except that the content popularity patterns can have much finer granularity than the traffic patterns due to the heterogeneity of content types. In addition, any user requesting specific contents typically wants to be associated with a BS which provides a high end-to-end service rate to the user and this association decision also depends on if the BS caches the corresponding

\footnotetext{
${ }^{1}$ According to a recent survey of Cisco, the mobile video traffic will account for more than $70 \%$ of total mobile traffic by 2020 [1].
}

contents. Therefore, content caching decisions must be made jointly with BS-user association decisions.

In this paper, we formulate the joint content caching and BS-user association problem to optimize the flow-level network performance, i.e., load balancing and end-to-end delay ${ }^{2}$ considering the spatio-temporal dynamic characteristics of content popularity patterns. Even though there have been some recent works dealing with joint content caching and BS-user association problems (e.g., see [2], [3]), they assumed fixed or general content popularity and did not capture different time scales of these network functions.

To that end, we address a flow-level BS-user association optimization problem in our design similarly to the previous works [6], [7], which aimed to achieve both efficient load balancing and delay minimization for all network flows without considerations of content caching in BSs. However, we would like to emphasize that our problem is more difficult to solve than those in the previous works. This is because the spatial heterogeneity of content popularity is incorporated in our optimization framework and the number of possible solutions of content caching at all BSs is much higher for our considered problem than those of BS on/off activation solutions in the previous work [6].

Because (i) finding an optimal solution directly for the considered joint content caching and BS-user association problems is challenging, and (ii) the time scales of flowlevel BS-user association and dynamics of content popularity patterns are very different, we take the time-scale separation design approach to develop the BS-user association and content caching control algorithms in our design framework. Specifically, we propose a short time-scale optimal BS-user association algorithm for a given content caching solution, which can be implemented in a distributed manner, and a long time-scale greedy content caching algorithm which guarantees to achieve $1-1 / e$ of an optimal objective value for most possible content caching sets. The contributions of this paper can be summarized as follows.

1) We formulate the joint content caching and BS-user association problems, which aim to optimize flow-level network performance, i.e., load balancing and end-to-

\footnotetext{
${ }^{2}$ It means delay from the closest content location, i.e., content servers or BSs which cache the contents, to each user location.
} 
end delay, considering the spatio-temporal dynamics of content popularity.

2) We propose an optimal BS-user association algorithm, namely OptUA for a given content caching solution. Moreover, we propose a low-complex greedy content caching algorithm, namely $G C C$, which operates on the long time scale while the OptUA algorithm is running on the short time scale. We show that the GCC algorithm guarantees to achieve a constant fraction of an optimal objective value for the most practical cases by exploiting submodular property of the objective function.

3) Via extensive simulation studies in heterogeneous cellular networks, we show that the proposed OptUA algorithm enables mobile users to be associated with BSs that cache the corresponding contents to optimize flow-level network performance. In addition, the proposed GCC algorithm considering the outcome of the optUA algorithm outperforms other general popularity based caching and random caching algorithms thanks to the exploitation of spatial heterogeneity of content popularity.

In the rest of this paper, we begin with the description of the system model in Section II. We present the joint content caching and BS-user association problems in Section III. In Section IV, we propose the OptUA algorithm and the GCC algorithm. We evaluate the performance of the proposed algorithms via extensive simulation in Section $\mathrm{V}$ followed by conclusions in Section VI.

\section{SySTEM MODEL}

We consider a cellular network which consists of multiple BSs with finite caching capacity. Let us denote the content set as $\mathcal{F}=\{1, \ldots, F\}$ where the file size of each content is denoted by $u$ (in bits). ${ }^{3}$ In addition, we assume that all contents are stored in their own servers distributed throughout the internet.

We consider a particular geographical region $\mathcal{L} \subset \mathbb{R}^{2}$ served by a set of BSs $\mathcal{B}$. Let $x \in \mathcal{L}$ denote some specific location of interest and $n \in \mathcal{B}$ denote the BS index. We assume that content requests for content $f$ arriving at any location $x$ according to an inhomogeneous Poisson point process with the average rate per unit area equal to $\lambda_{f}(x), \forall f \in \mathcal{F}$. Then, the corresponding traffic load can be expressed as $\gamma_{f}(x) \doteq \lambda_{f}(x) u<\infty, \forall f \in \mathcal{F}, x \in \mathcal{L}$. It can be observed that this traffic model enables us to capture spatial heterogeneity in dynamic content requests.

Let us denote caching decision variables as $\boldsymbol{I} \doteq\left\{I_{f}^{n} \mid \forall n \in\right.$ $\mathcal{B}, \forall f \in \mathcal{F}\}$ where $I_{f}^{n}=1$ if content $f$ is cached in BS $n$, otherwise, $I_{f}^{n}=0$. Equivalently, we also introduce a vector $\mathcal{S}=\left[\mathcal{S}^{n}, n \in \mathcal{B}\right]$ whose set elements represent the sets of cached contents in different BSs where $\mathcal{S}^{n}$ denotes the set of cached contents in BS $n^{4}$. Because the time scale for updating content caching decisions must correspond to the time scale over which the content popularity varies, e.g.,

\footnotetext{
${ }^{3}$ Our design can be extended to the scenario with heterogeneous content size.

${ }^{4}$ Hereafter, we use $\boldsymbol{I}$ and $\mathcal{S}$ interchangeably to describe the content caching decisions.
}

few hours, we assume the time scale for updating content caching decision is much longer than that for making BSuser association decisions. With these defined caching decision variables, finite caching capacity constraints at individual BSs can be expressed as follows:

$$
\sum_{f} u I_{f}^{n} \leq T_{n}, \quad \forall n \in \mathcal{B},
$$

where $T_{n}$ denotes the caching capacity of BS $n$.

For a given caching solution $\mathcal{S}$ in all BSs, the average endto-end data rate from $\mathrm{BS} n \in \mathcal{B}$ or original content server of each content to location $x$ for content $f$ can be calculated as $c_{f}^{b}(n, x) I_{f}^{n}+c_{f}^{e}(n, x)\left(1-I_{f}^{n}\right)$ where $c_{f}^{b}(n, x)$ denotes the average wireless data rate for content $f$ between BS $n$ and a user at location $x$ if the content is cached at the BS $n$, and $c_{f}^{e}(n, x)$ denotes average end-to-end data rate from the closest content server which stores content $f$ to user at location $x$ via BS $n$ where $c_{f}^{e}(n, x)=\min \left(c_{f}^{s}(n), c_{f}^{b}(n, x)\right)$ where $c_{f}^{s}(n)$ expresses the backhaul data rate to support data delivery from the content server of content $f$ to BS $n$.

Note that $c_{f}^{b}(n, x)$ and $c_{f}^{s}(n)$ denote the average rates where the effects of wireless fading or dynamic inter-cell interference are averaged out. This can be justified by the fact that the time intervals over which caching and BS-user association control decisions are made are large (i.e., a few minutes to hours). These assumptions have also been adopted in previous loadbalancing works [8], [9].

Using these definitions, the load density $\varrho_{f}^{n}(x)$ can be defined as $\varrho_{f}^{n}(x)=\frac{\gamma_{f}(x)}{c_{f}^{b}(n, x) I_{f}^{n}+c_{f}^{e}(n, x)\left(1-I_{f}^{n}\right)}$, which represents the fraction of time required to deliver content load $\gamma_{f}(x)$ of content $f$ from BS $n$ to a user at location $x$. In addition, we introduce a routing function $\boldsymbol{p}=\left\{p_{f}^{n}(x) \mid \forall n \in \mathcal{B}, \forall x \in\right.$ $\mathcal{L}, \forall f \in \mathcal{F}\}$ where $p_{f}^{n}(x)$ denotes the probability that a user flow of content $f$ at location $x$ is associated with $\mathrm{BS} n$. Intuitively, $p_{f}^{n}(x)$ can be interpreted as the time fraction that a flow of content $f$ arriving at location $x$ is routed to BS $n$. It turns out that the optimal $p_{f}^{n}(x)$ will be either 1 or 0 , i.e., deterministic routing (or BS-user association) is the solution of our optimization problem defined later in $(\mathbf{P})$.

We now provide the definition of content load feasibility for each BS in the following.

Definition 1. (Feasibility): The set $\mathcal{H}(\boldsymbol{I})$ of feasible content loads in each $B S \rho=\left(\rho_{1}^{1}, \ldots, \rho_{|\mathcal{F}|}^{|\mathcal{B}|}\right)$ for a given content caching solution for all BSs $\boldsymbol{I}$ can be defined as

$$
\begin{gathered}
\mathcal{H}(\boldsymbol{I})=\left\{\rho \mid \rho_{f}^{n}=\int_{\mathcal{L}} \varrho_{f}^{n}(x) p_{f}^{n}(x) d x, \forall n \in \mathcal{B}, \forall f \in \mathcal{F},\right. \\
0 \leq \rho_{f}^{n} \leq 1-\epsilon, \forall f \in \mathcal{F}, \forall n \in \mathcal{B}, \\
\sum_{n \in \mathcal{B}} p_{f}^{n}(x)=1, \forall x \in \mathcal{L}, \forall f \in \mathcal{F}, \\
\left.0 \leq p_{f}^{n}(x) \leq 1, \forall n \in \mathcal{B}, \forall x \in \mathcal{L}, \forall f \in \mathcal{F}\right\}
\end{gathered}
$$

where $\epsilon$ is an arbitrarily small positive constant. Note that any feasible BS and content load $\rho$ has the corresponding routing probability vector $p(x)=\left(p_{1}^{1}(x), \ldots, p_{|\mathcal{B}|}^{|\mathcal{F}|}\right)$ for all $x \in \mathcal{L}$. 
Lemma 1. The feasible set $\mathcal{H}(\boldsymbol{I})$ is convex.

Proof. It can be easily proved using typical definition of convex function and consideration of heterogeneity of content popularity. The detailed proof is presented in our technical report [10].

\section{Problem Statement and Two Time-Scale DESIGN APPROACH}

\section{A. Problem Formulation}

Our design problem (P) aims to find an optimal set of joint cached contents in each BS and BS-user association solution (i.e., BS load $\rho$ ) which minimizes the flow-level cost function as follows:

$$
\text { (P): } \min _{\rho, \boldsymbol{I}}\left\{A_{\alpha}(\rho, \boldsymbol{I}) \mid \rho \in \mathcal{H}(\boldsymbol{I})\right\}, \quad \text { s.t. constraint (1). }
$$

The flow-level cost function $A_{\alpha}(\rho, \boldsymbol{I})$ is designed based on $\alpha$-delay performance function in [6]. However, we additionally take into account content diversity and backhaul data rate in the original $\alpha$-delay performance function as follows:

$$
A_{\alpha}(\rho, \boldsymbol{I})=\left\{\begin{array}{l}
\sum_{n \in \mathcal{B}} \sum_{f \in \mathcal{F}} \frac{\left(1-\rho_{f}^{n}\right)^{1-\alpha}-1}{\alpha-1}, \text { if } \alpha \neq 1, \\
\sum_{n \in \mathcal{B}} \sum_{f \in \mathcal{F}} \log \left(\frac{1}{1-\rho_{f}^{n}}\right), \text { if } \alpha=1,
\end{array}\right.
$$

where $\alpha \geq 0$ denotes a tradeoff parameter between load balancing and minimization of total BS and content load.

When $\alpha=0$ (rate-optimal), $A_{0}(\cdot)$ becomes $\sum_{n} \sum_{f} \rho_{f}^{n}$. When $\alpha=2$ (end-to-end delay-optimal), $A_{2}(\cdot)$ becomes $\sum_{n} \sum_{f} \frac{\rho_{f}^{n}}{1-\rho_{f}^{n}}$, which is equal to the total average number of serviced contents in the system if we consider the system as an M/GI/1 multi-class processor sharing system [11]. From the Little's law, minimizing $A_{2}(\cdot)$ is equivalent to minimizing the average end-to-end delay ${ }^{5}$ per each content request. Other cases and details are presented in our technical report [10].

\section{B. Time Scale Separation Approach}

It is very difficult to solve the general problem $(\mathbf{P})$ because the BS-user association problem and content caching problem are tightly intertwined with each other. To facilitate the algorithm design, we assume a time-scale separation design rationale exploiting the fact that content request arrival and departure, and the corresponding BS-user association decisions occur on a much faster time scale compared to the time scale over which content caching decisions are updated.

Since the time-scale for BS content caching controls is similar to that of the content request pattern variations, this time scale is apparently much longer than that for flow-level BS-user association. That is, the time scale can be separated between the BS-user association and the content caching decisions. Leveraging this time scale separation, the problem (P) can be solved by decomposing it into two problems. In particular, the solution of content caching problem is updated on the slower time scale than the BS-user association decision. We now discuss how to tackle the BS-user association and content caching problems.

\footnotetext{
${ }^{5}$ Hereafter, we call end-to-end delay as just delay for simplicity.
}

BS-user association problem: For a given caching solution (i.e., the content caching vector $\boldsymbol{I}$ ), problem (P) becomes the following short time-scale problem:

\section{BS-User Association Problem [P-UA]:}

$$
\min _{\rho}\left\{A_{\alpha}(\rho, \boldsymbol{I}) \mid \rho \in \mathcal{H}(\boldsymbol{I})\right\} .
$$

Solving this problem is equivalent to find the BS-user association set $\boldsymbol{p}=\left\{p_{f}^{n}(x) \mid n \in \mathcal{B}, x \in \mathcal{L}, f \in \mathcal{F}\right\}$.

Content caching problem: This longer time-scale problem aims to find a set of contents to be cached in each BS as follows:

\section{Content Caching Problem [P-CC]:}

$$
\min _{\boldsymbol{I}}\left\{G(\boldsymbol{I}) \doteq \min _{\rho \in \mathcal{H}(\boldsymbol{I})} A_{\alpha}(\rho, \boldsymbol{I})\right\}, \quad \text { s.t. constraint (1) }
$$

where the objective function $G(\boldsymbol{I})$ corresponds to the objective function in [P-UA] with the optimal BS-user association solution.

\section{BS-User ASSOCIATION AND CONTENT CACHING ALGORITHMS}

\section{A. Short Time Scale BS-User Association Algorithm}

This short time scale BS-user association can be similarly derived as in [6] except that content diversity is considered in our design.

Optimal BS-user association policy. We denote the optimal BS load vector as $\rho^{*}=\left(\rho_{1}^{1^{*}}, \ldots, \rho_{|\mathcal{F}|}^{\mid \mathcal{B}}\right)$, which is the solution of the problem [P-UA], and denote the optimal BS-user association solution for content $f$ at location $x$ as $n_{f}^{*}(x)$. We describe the optimality condition of this problem, which is used to develop an optimal BS-user association policy, in the following theorem.

Theorem 1. If the problem [P-UA] is feasible, then the optimal $B S n_{f}^{*}(x)$ with which a user at location $x$ requesting content $f$ is associated is given by

$$
\begin{array}{r}
n_{f}^{*}(x)=\underset{m \in \mathcal{B}}{\arg \max } \frac{c_{f}^{b}(m, x) I_{f}^{m}+c_{f}^{e}(m, x)\left(1-I_{f}^{m}\right)}{\left(1-\rho_{f}^{m, *}\right)^{-\alpha}}, \\
\forall x \in \mathcal{L}, \forall f \in \mathcal{F} .
\end{array}
$$

Proof. This theorem can be proved using the standard optimization theory. The proof is presented in our technical report [10].

\section{B. Long Time Scale Content Caching Algorithm}

Rationale of the content caching algorithm. The objective function $G(\rho, \boldsymbol{I})$ is a non-convex and discontinuous function because of discrete decision variables $\boldsymbol{I}$. Hence, this content caching problem is a combinatorial problem with $O\left(2^{|\mathcal{B}||\mathcal{F}|}\right)$ possible solutions, which is difficult to solve when the numbers of contents and BSs are large. Fortunately, the objective function $G(\rho, \boldsymbol{I})$ is convex in $\rho$ and its optimal solution can be determined for a given $\boldsymbol{I}$ or $\mathcal{S}$.

Note that the function $G(\boldsymbol{I})=G(\mathcal{S})=\min _{\rho} A(\rho, \boldsymbol{I}), \rho \in$ $\mathcal{H}(\boldsymbol{I})$ is obtained by applying the OptUA policy given in Section IV-A. Moreover, the function $G(\mathcal{S})$ is a surjective 


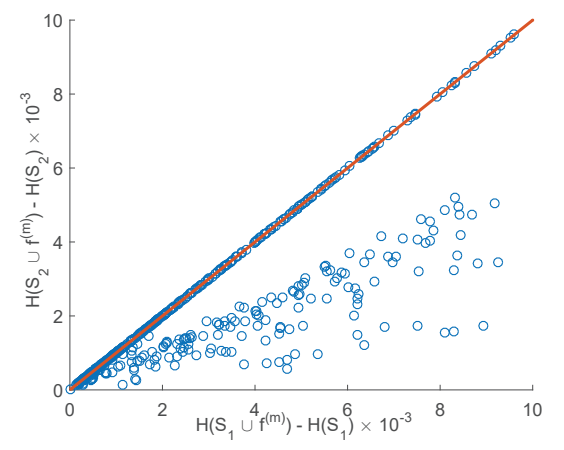

Fig. 1: Verification for the submodular set function with $\alpha=2$ where $S_{1} \subset S_{2}$.

function, i.e., the exact optimal value of $G(\mathcal{S})$ exists for all possible caching sets $\mathcal{S}^{n}$ for all $n \in \mathcal{B}$. Then, we can consider an equivalent objective with $(\mathbf{P})$ in the following:

$$
\text { (IP): } \max _{\mathcal{S}} H(\mathcal{S}), \quad \text { s.t. constraint (1), }
$$

where $H(\mathcal{S})=G(\phi)-G(\mathcal{S})$ where $\phi$ means empty set. If the objective function $H(\mathcal{S})$ in (IP) is a non-decreasing submodular set function, [12] showed that a known modified greedy algorithm achieves $1-1$ /e performance of the optimal objective function value. To exploit this result, we verify whether the function $H(\mathcal{S})$ is non-decreasing and submodular set function or not. The definition of the submodular set function is given as follows.

Definition 2. A real-valued set function $g(\cdot)$, defined on subsets of finite sets $\mathcal{F}$ for all BSs is named submodular set function if it satisfies following condition for all $\mathcal{S} \subseteq \mathcal{S}^{\prime}$ and for all $f^{(n)} \in \mathcal{F} \backslash \mathcal{S}^{n^{\prime}}, \forall n \in \mathcal{B}$,

$$
g\left(\mathcal{S} \cup\left\{f^{(n)}\right\}\right)-g(\mathcal{S}) \geq g\left(\mathcal{S}^{\prime} \cup\left\{f^{(n)}\right\}\right)-g\left(\mathcal{S}^{\prime}\right) .
$$

It can be verified that the objective function $H(\mathcal{S})$ is a submodular set function for most feasible caching sets for other values of $\alpha$, i.e., $\alpha=2,3,4, \ldots$, via numerical tests (the case with $\alpha=2$ is illustrated in Fig. 1). In summary, a known greedy algorithm in [12] which is slightly different from our proposed algorithm described in the following guarantees to achieve $1-1 / e$ of the optimal objective function value $H\left(\mathcal{S}^{*}\right)$ for most feasible caching sets.

Inspired by these facts, we propose a greedy content caching algorithm in the following.

Greedy content caching (GCC) algorithm. We first describe a greedy content caching algorithm, called $G C C$, that iteratively caches contents so that it can achieve the most reduction in the objective value.

The main idea of the GCC algorithm is to iteratively cache contents in BSs one by one considering the global objective reduction for all contents in all BSs until caching capacities in all BSs are completely filled up. As discussed before, content caching and corresponding BS-user association decisions are coupled over all BSs. Hence, we need to carefully cache contents considering the current content caching and BS-user association status in all BSs.

\section{Greedy Content Caching Algorithm (GCC)}

1: Initialization: $\mathcal{S}^{n}=\phi, \quad \forall n \in \mathcal{B}$

2: While (caching capacity is not completely filled up for any BSs, i.e., $\left|\mathcal{S}^{n}\right|<\left\lfloor T_{n} / u\right\rfloor$, for any $n \in \mathcal{B}$ )

3: $\quad$ For all $n \in \mathcal{B}$,

4: $\quad$ If $\left|\mathcal{S}^{n}\right|=\left\lfloor T_{n} / u\right\rfloor$, then $\mathcal{B} \leftarrow \mathcal{B} \backslash n$

5: $\quad$ End For

6: Calculate

$$
\begin{array}{r}
M_{G}(f(m))=G\left(\left[\mathcal{S}^{n}, \forall n \in \mathcal{B} \backslash m\right] \cup\left\{\mathcal{S}^{m} \cup\{f(m)\}\right\}\right), \\
\forall f(m) \in \mathcal{F} \backslash \mathcal{S}^{m}, \quad \forall m \in \mathcal{B}
\end{array}
$$

where OptUA policy is applied in $G(\cdot)$.

7: $\quad$ Find the content

$$
f^{*}\left(m^{*}\right)=\underset{f(m) \in \mathcal{F} \backslash \mathcal{S}^{m}, \forall m \in \mathcal{B}}{\arg \min } M_{G}(f(m))
$$

8: $\quad$ Cache $f^{*}\left(m^{*}\right)$ in the BS $m^{*}$, i.e., $\mathcal{S}^{m} \leftarrow \mathcal{S}^{m} \cup m$.

9: End While

In steps 3, 4 and 5 of the GCC algorithm, we verify whether the caching capacity of each BS is completely filled up or not to reduce the searching space and calculation efforts of the greedy caching metrics. In step 6, we calculate the caching metrics $M_{G}(f(m))$ for all uncached contents in all BSs except for BSs whose caching capacities have been filled up. In steps 7 and 8 , we find content $f$ in BS $m$ which minimizes the greedy metric $M_{G}(f(m))$ and cache this "best" content $f$ in BS $m$. Note that in each iteration, the GCC algorithm only caches one content in one BS which minimizes the caching metric. Therefore, if the maximum number of contents which can be cached in each BS is 10 and the number of BSs is 10 , then the maximum number of iterations performed by the GCC algorithm is $10 \times 10=100$.

We believe that the proposed GCC algorithm brings a significant performance enhancement because it attempts to cache the best content in each step using the metric, which is the value of the $G(\cdot)$ obtained after optimizing the BS-user association decision variables. This algorithm also exploits the structure of the objective function and caching constraints in (P), which accounts for the heterogeneity of content popularity, and current caching and BS-user association status in all BSs to make content caching decisions.

\section{Performance Evaluation}

Simulation setup. We evaluate the performance of the proposed joint content caching and BS-user association algorithms via extensive simulations under various configurations. The considered network topology consists of five macro BS and five micro BSs in $2 \times 2 \mathrm{~km}^{2}$ as shown in Fig. 2. We use mean transmission powers for BSs given in [13], which are $20 \mathrm{~W}$ and $1 \mathrm{~W}$ for macro and micro BSs, respectively. We consider 100 different contents with the same size (10Mbytes). Moreover, we assume that each BS can cache up to 10 contents. Other parameters for the simulations are set according to the IEEE 


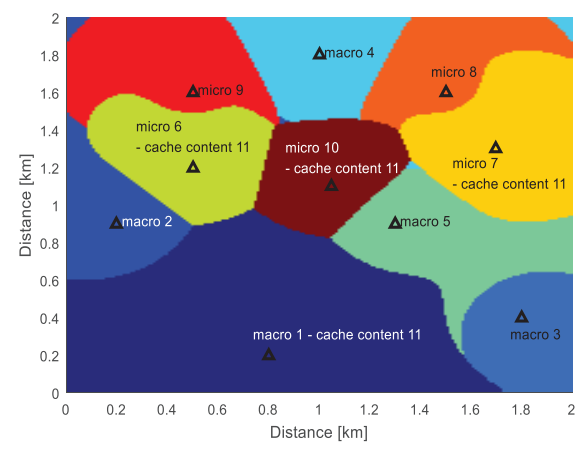

(a) OptUA (Content popularity-aware association).

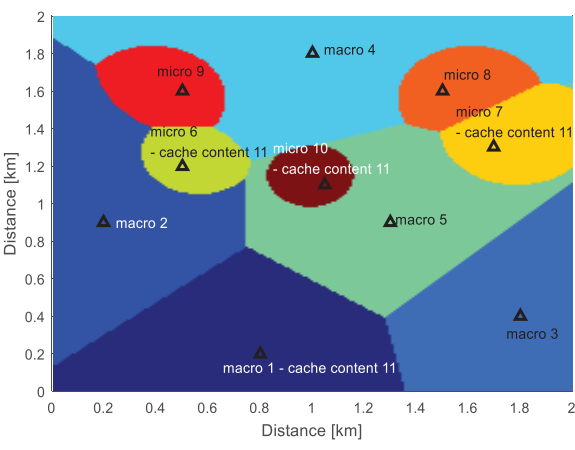

(b) FLA (Traffic-aware association).

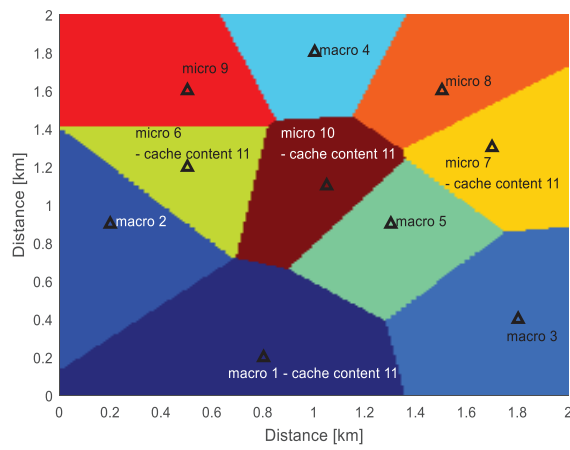

(c) NBA (Near BS association).

Fig. 2: Snapshots of cell coverage for content 11 by different user association algorithms with random caching policy $(\alpha=2)$.

$802.16 \mathrm{~m}$ evaluation methodology document [14]. In addition, we assume contents in each BS receive the same fraction of scheduling time, hence, $c_{f}^{b}(n, x)$ is the same for all contents. The data rate from original content servers to each BS is set to be $100 \mathrm{kbps}-1 \mathrm{Mbps}$ which are the average values drawn from a real YouTube dataset [15].

We consider two objective functions corresponding to different values of $\alpha$ in our simulation study: 1) the average system load per each content in a BS $(\alpha=0)$ and 2) the average content service delay $(\alpha=2)$. In addition, we compare the performance of the proposed caching and BS-user association algorithms with that of the following algorithms: 1) flowlevel BS-user association (FLA) in [6] and 2) nearest BS user association (NBA) under two baseline caching policies: random (RAND) caching and general popularity (GP) based caching. The RAND caching policy caches contents in each BS in the random manner until the caching capacity is filled up while the GP based caching policy uses the general popularity, i.e., all BSs assume the same popularity profile for all contents. The FLA algorithm does not consider the heterogeneity of content popularity over different contents, but takes into account only the total arrival rate, i.e., $\sum_{f} \lambda_{f}(x)$ for each location $x$. Moreover, each user is associated with the nearest BS under the NBA algorithm.

Simulation results. We carry out the simulations under two different content request scenarios: 1) homogeneous content request distribution, i.e., $\lambda_{f}(x)=\lambda_{f}$ for all $f \in \mathcal{F}$ and for all $x \in \mathcal{L}$, where $\lambda_{f}$ follows Zipf distribution [16] for all contents, and 2) heterogeneous content request distribution, i.e., $\lambda_{f}(x)$ varies from location to location and content to content. We assume the Gaussian distribution with mean $\lambda_{f}$ and variance $\lambda_{f}^{2}$. We discuss the simulation results and the key observations in the following.

Impact of caching-aware BS-user association on the cell coverage. Fig. 2 depicts snapshots of cell coverage for one specific content by different BS-user association algorithms with RAND caching policy when $\alpha=2$. In the FLA algorithm, many users prefer to be associated with macro BSs to attain higher data rates whereas all users are associated with their closest BSs in the NBA algorithm. Moreover, in the OptUA algorithm, the cell coverages of BSs which have the corresponding content (i.e., content 11) are larger than that due to other BS-user association algorithms since the OptUA efficiently exploits caching status information in all BSs to achieve the best objective value.

We now evaluate the performance of the proposed GCC algorithm in conjunction with different BS-user association algorithms and other content caching algorithms in conjunction with the proposed OptUA algorithm.

Impact of the backhaul data rate. Figs. 3.(a),(b) depict the average system load per content in each BS and the average service delay per content request versus the backhaul data rate. As the backhaul data rate decreases, the GCC algorithm attains higher performance compared to the other caching policies in both performance metrics. In particular, the OptUA+GCC attains 25\% lower average delay than the OptUA+GP and 66\% lower average delay than the Opt+RAND where the backhaul data rate is $0.1 \mathrm{Mbps}$. This is because the GCC algorithm takes into account the spatial heterogeneity of content popularity in making caching decisions; hence, more content requests are serviced from BSs that cache the corresponding contents under the GCC algorithm whereas other caching algorithms tend to retrieve requested contents from their original content servers more frequently and use backhaul links.

Impact of BS-user association algorithms. As shown in Fig. 3.(a),(b), the proposed GCC algorithm attains the best performance when it is jointly used with the OptUA algorithm compared to scenarios where other BS-user association algorithms are employed. This is because the GCC algorithm takes into account the objective function value $G(\cdot)$, which is a function of the optimal BS-user association solution ${ }^{6}$ to make content caching decisions. Moreover, the FLA algorithm with GCC algorithm outperforms the NBA algorithm with GCC algorithm in the inhomogeneous content request distribution scenario because under the FLA algorithm, BS-user association decisions account for the spatial variability of traffic distribution.

Impact of spatial heterogeneity of content popularity. Fig. 3(c) depicts the delay performance of the OptUA+GCC algorithms compared to the OptUA+GP algorithms as a function of a

\footnotetext{
${ }^{6}$ The optimal BS-user association solution can be obtained by the OptUA algorithm.
} 


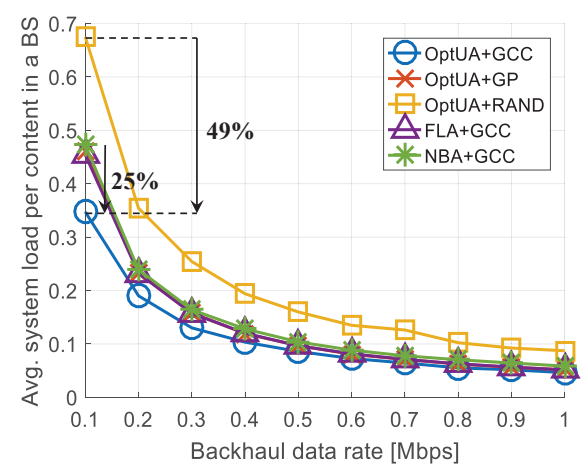

(a) $\alpha=0$ : avg. system load per content in a BS.

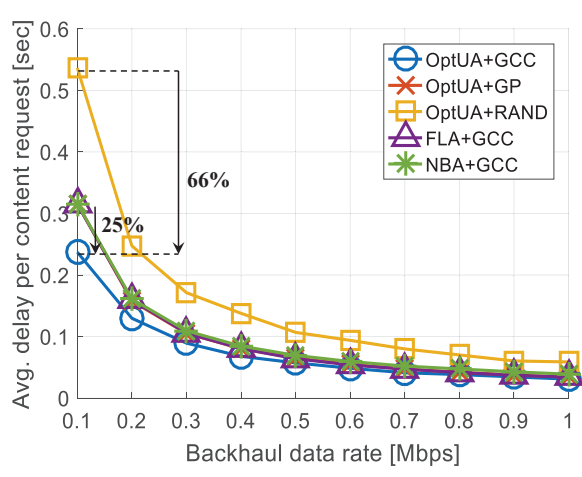

(b) $\alpha=2$ : avg. delay per content request.

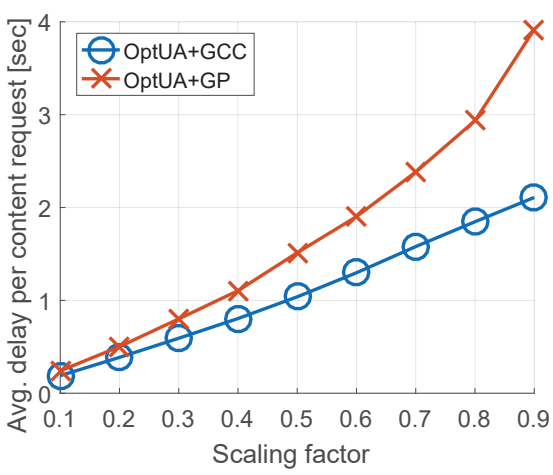

(c) Impact of spatial heterogeneity of popularity.

Fig. 3: Performance of proposed BS-user association and content caching algorithms in heterogeneous content request.

scaling factor which captures the degree of spatial heterogeneity of content popularity. As the scaling factor increases, i.e., increasing spatial heterogeneity of the content popularity, the performance gap between OptUA+GCC and OptUA+GP becomes larger. We can see that average service delay per content request due to the OptUA+GP is almost twice of that when the scaling factor is equal to 0.9 .

\section{Concluding Remark}

In this paper, we have studied the dynamic content caching and BS-user association problems considering the spatial variation of content popularity. To tackle this challenging joint problem, we have proposed an online optimal BS-user association algorithm operating on the short time scale for a given content caching solution, and a greedy content caching algorithm operating on the long time scale. Through extensive simulations in the heterogeneous cellular networks, we have showed the performance gains of the proposed algorithms against other existing algorithms in two performance metrics, namely the average service delay and the average system load per content in each BS.

\section{ACKNOWLEDGEMENT}

This work was supported by grants from The Natural Sciences and Engineering Research Council of Canada (NSERC). Also, this project has received funding from the European Union's Horizon 2020 research and innovation programme under the Marie Skłodowska-Curie grant agreement No 713567.

\section{REFERENCES}

[1] Cisco, San Jose, CA, "Cisco Visual Networking Index: Globel Mobile Data Traffic Forecast Update, 2015-2020." [Online]. Available: http://www.cisco.com/c/en/us/solutions/collateral/service-provider/ visual-networking-index-vni/mobile-white-paper-c11-520862.pdf

[2] Y. Wang, X. Tao, X. Zhang, and G. Mao, "Joint caching placement and user association for minimizing user download delay," IEEE Access, vol. 4, pp. 8625-8633, Nov. 2016.

[3] B. Dai and W. Yu, "Joint user association and content placement for cache-enabled wireless networks," in Proc. of IEEE ICASSP, Shanghai, China, Mar. 2016, pp. 1-5.

[4] H. Pedersen and S. Dey, "Enhancing mobile video capacity and quality using rate adaptation, RAN caching and processing," IEEE/ACM Trans. on Netw., vol. 24, no. 2, pp. 996-1010, Apr. 2016.
[5] J. Kwak, Y. Kim, L. Le, and S. Chong, "Hybrid content caching for low end-to-end latency in cloud-based wireless networks," in Proc. of IEEE ICC, Jun. 2017, pp. 1-6.

[6] K. Son, H. Kim, Y. Yi, and B. Krishnamachari, "Base station operation and user association mechanisms for energy-delay tradeoffs in green cellular networks," IEEE JSAC, pp. 1525-1536, Sep. 2011.

[7] H. Kim, G. de Veciana, X. Yang, and M. Venkatachalam, "Distributed $\alpha$-optimal user association and cell load balancing in wireless networks," IEEE/ACM Trans. on Netw., vol. 20, no. 1, pp. 177-190, Feb. 2012.

[8] A. Sang, M. Madihian, X. Wang, and R. Gitlin, "Coordinated load balancing, handoff/cell-site selection, and scheduling in multi-cell packet data systems," in Proc. of ACM MobiCom, Sep. 2004, pp. 301-314.

[9] K. Son, S. Chong, and G. Veciana, "Dynamic association for load balancing and interference avoidance in multi-cell networks," IEEE Trans. on Wireless Commun., vol. 8, no. 7, pp. 3566-3576, Jul. 2009.

[10] J. Kwak, L. Le, and X. Wang, "Two time-scale content caching and user association in 5G heterogeneous networks," Technical report. [Online]. Available: https://www.dropbox.com/s/ puu3c50gufiwo5r/TechreportKwak17.pdf?dl=0

[11] J. Walrand, An introduction to queueing networks. Prentice Hall, 1998.

[12] M. Sviridenko, "A note on maximizing a submodular set function subject to knapsack constraint," Operations Research Letters, pp. 41-43, 2004.

[13] Ofcom, "Mobile phone Base Station Database." [Online]. Available: http://www.sitefinder.ofcom.org.uk/

[14] H. Kim, X. Yang, and M. Venkatachalam, "Handover and load balancing rules for 16m," IEEE C802.16m-09/0136rl, pp. 1024-1037, Jan. 2009.

[15] Y. G. M. Zink, K. Suh and J. Kurose, "Characteristics of YouTube network traffic at a campus network - Measurements, models, and implications," Elsevier Computer Networks, pp. 501-514, Mar. 2009.

[16] Z. Zhao, M. Peng, Z. Ding, W. Wang, and H. Poor, "Cluster content caching: An energy-efficient approach to improve quality of service in cloud radio access networks," IEEE JSAC, vol. 34, no. 5, pp. 1207-1221, May 2016. 